\title{
Decisiones sobre las personas: Un análisis psicosocial de los procesos clasificatorios
}

\author{
JULIO F. VILLEGAS \\ Pontificia Universidad Católica de Chile

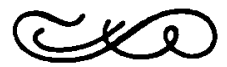

\section{Resumen}

El trabajo presenta una nuea perspectiva para el examen de los procesos de percepción interpersonal. Se destaca tanto el interés psicosocial, como las posibilidades clásicas de explicación para estos eventos, ofreciéndose al mismo tiempo una explicación alternativa basada en una concepción amplia de la toma de decisiones.

Finalmente, se reporta un ejemplo de investigación empirica en proceso, referida especificamente al tema.

\section{Abstract}

The aim of this paper is to show a new approach to the exploration of interpersonal perception processes. It points out the psychosocial interest as well as the traditional possibilities of explaining this events and at the same time, it offers an alternative explanation based on a wide conception of decision making.

Finally, it reports an example of empirical research under way, specifically related to the subject.

Dirección del autor: Departamento de Psicología Básica. Escuela de Psicología. Pontificia Universidad Católica de Chile. Casilla 114-D. Santiago. Chile.

Notas: Algunos de los contenidos desarrollados en este trabajo, forman parte del proyecto de la tesis del autor para el Magister Sceitnarium en Psicología Social, presentado en la Universidad Central de Venezuela (1980). 


\section{INTRODUCCION}

Los procesos de interacción han sido temática recurrente de la investigación científica en psicología social. Una somera revisión de la literatura ofrece la posibilidad de concebirlos como origen de la influencia social, de las relaciones de poder o de la comunicación persuasiva; como antecedentes y locus de expresión para las diferentes modalidades de la percepción y aprendizaje sociales; como las bases para el desarrollo, mantenimiento y cambio de creencias, actitudes, valores, roles, estereotipos, prejuicios, etc. Por otra parte, el examen de los procesos de interacción social puede orientarse a las relaciones entre los organismos, como también a la interdepedencia de éstos con respecto a las variables de su contexto, cubriendo así prácticamente todos los eventos que se incluyen en el campo de la psicología social.

La impresionante riqueza de fenómenos asociados a la interacción, incluye preferentemente manifestaciones de la percepción social y, específicamente, de la percepción interpersonal. En este último sentido y para los propósitos de este trabajo, interesa particularmente destacar que las relaciones entre los seres humanos propician adscripciones a una serie de categorías dando lugar a lo que hemos llamado procesos de clasificación social. En otras palabras, cuando describimos a una persona decimos, por ejemplo, que ella nos parece de un determinado nivel económico, social o cultural; que su conducta verbal nos revela ciertos intereses; que su conducta expresiva nos indica algunos hábitos o prácticas o que finalmente, nos han impresionado determinadas características o rasgos de su personalidad.

La importancia psicosocial de los procesos clasificatorios, puede expresarse a lo menos en los siguientes términos:

1. Clasificar a una persona, digamos en una categoría ocupacional, puede generar expectativas respecto de una serie de patrones considerados apropiados o socialmente aceptados. Friedland, Crockett y Laird (1973), reportan que la categoría "trabajador social" despierta expectativas referidas a cualidades y comportamientos expresivos tales como, mejores amigos, más tratables, más agradables, etc., en comparación a la categoría «ingeniero". Cabe destacar que la generación de las expectativas en este caso, es independiente del sexo de los que ocupan estas categorías.

2. Las expectativas así generadas, pueden repercutir en la producción de consecuencias para la persona que ha sido objeto de una clasificación, las que a su vez, pueden manifestarse a dos niveles.

2.1. En un plano afectivo-emocional, en la medida que las expectativas se acompañen de reacciones evaluativas como pueden ser las de atracción o rechazo, simpatía o antipatía, etc. (Santoro, 1978).

2.2. En un plano conductual, como lo señalan Kleck, Ono y Hastorff (1966), al examinar las variaciones producidas en una interacción diádica, como consecuencia de la presentación de uno de los sujetos, como sufriendo de una gravísima enfermedad. Asimismo, Rosenthal y Jacobson (1968) crearon deliberadamente expectativas a un grupo de maestros respecto a las capacidades de sus estudiantes, para demostrar cómo aquéllas pueden afectar incluso la evaluación del rendimiento escolar. Este hallazgo ha pasado 
a ser conocido como el «efecto Pigmalión" y es de hacer notar que en Latinoamérica, Orantes (1979) ha trabajado en la misma línea. Son también suficientemente conocidas las formulaciones sobre las consecuencias sociales de la estigmatización presentada por Becker (1971) en sus estudios sobre la desviación, término que preferimos reemplazar denotativamente por disidencia.

\section{Posibilidades psicosociales para la explicación}

Lo expresado hasta ahora hace comprensible el notable esfuerzo de investigación científica dedicada al examen de los mecanismos, procedimientos y/o estrategias a través de las cuales se produce la clasificación social de las personas. $\mathrm{Al}$ respecto, podemos afirmar que se dispone tanto de trabajos empíricos como de formulaciones teóricas que hacen referencias a:

1. La formación de impresiones, proceso a través del cual las personas organizan cognoscitivamente contenidos específicos, en estructura coherentes y afectivamente polarizadas acerca de los demás (Livesley y Bromley, 1973).

Los diversos aspectos de este proceso pueden encontrarse en Rommetveit (1960), Sharauger (1967), Secord y Backman (1964); Berkowitz (1974), Tajfel (1969), Jahoda (1956); Allport (1954) y muchos más.

2. La asignación de rasgos, proceso en el cual las personas infieren la existencia de ciertas características en los demás. En esta área son conocidos los trabajos de Asch (1946); Wishner (1960); Warr y Knapper (1965); Schneider (1973); Anderson (1965); Willis (1960); Rokeach y Rothman (1965), para señalar sólo algunos.

3. La formación de constructos personales originalmente planteada por Kelly (1955), señalando que la percepción de la realidad -incluidas las personas- se basa en una representación psicológica que hacemos del ambiente. Esta formulación asume un reconocimiento para una especie de tendencia de los seres humanos hacia comportarse como un científico que inventa, construye y desarrolla "teorías" que le permitan conocer, explicar y/o predecir la realidad en la que permanentemente se desenvuelve.

4. Los modelos de inferencia o las secuencias y pasos que anteceden la inferencia de las causas e intenciones del comportamiento y que han merecido trabajos como los de Jones y Davis (1965); Sarbin, Taft y Bailey (1960); Tagiury (1969), entre los de mayor importancia.

5. Los procesos de atribución, referidos a la adjudicación de intenciones y rasgos que sirven como información para otorgar disposiciones a las personas y causas para sus conductas. Se sostiene que este proceso tiene lugar en función de explicar y anticipar el comportamiento de los demás. En esta línea, pueden mencionarse los trabajos de Kelley (1967); Kruglansky (1975); Nisbett y Borgide (1975); Jones y Aronson (1973); Lerner (1971); Baron, Griffitt y Byrne (1974); Arkin y Duval (1975), junto a otros muchos.

6. Los procesos de etiquetamiento social de la agresión, respecto de los cuales Bandura (1973) señala que una respuesta puede o no ser clasificada como agresiva en consideración de factores inherentes a su fuerza, duración o consecuencias producidas; en función de factores asociados a las características de los emisores, de los observadores de las víctimas, etc. Qui- 


\section{8}

zá con el mismo razonamiento, Fishbein (1979) sostiene que la agresión antes que un comportamiento propiamente tal, debe ser considerada definitivamente como una categoría conductual.

Determinar relaciones de correspondencia, complementariedad o incompatibilidad entre todos y cada uno de estos procesos, es una tarea atractiva que, sin embargo, excede a nuestros intereses y posibilidades inmediatas. Lo que podemos afirmar es que existe una evidencia empírica sustancial que hace posible establecer que en la interacción social las personas clasifican a los demás en un variado conjunto de categorías recurriendo a sus impresiones, inferencias, constructos personales, atribuciones, etc.

\section{Una posibilidad de explicación alternativa}

Aunque pudiera subyacer a los procesos reseñados más arriba, nos parece sugerente concebir explícitamente a la clasificación social de las personas como una variación de los procesos de toma de decisiones, en el sentido más amplio que pueda dársela. En síntesis, puede deducirse que se trata de determinar o decidir la pertenencia de uno o más sujetos a categorías o agrupaciones cualesquiera.

Parece fundamental establecer que al hablar de una toma de decisiones para los procesos clasificatorios, lo hacemos en un sentido amplio en la medida que por una parte, no excluya la posibilidad de asumir el valor de una decisión implícita y por la otra, las circunstancias de sustentarse en elementos subjetivos o irracionales. Por lo demás, la psicológica ha sido considerada como una línea muy prometedora en el desarrollo contemporáneo de nuestra disciplina (Rodrígues, 1979).

Ahora bien, cualquier toma de decisiones exige o requiere de una cierta información que sirve como base para sustentarla, sea a priori o a posteriori. En este orden de cosas, las fuentes a las que usualmente puede recurrirse parecen ser las siguientes:

1. Eventualmente, las personas pueden asumir que la decisión se sustentará sobre la base del respeto a la autoclasificación que los sujetos hagan de sí mismos. En otras palabras, se decide que una persona pertenece a una categoría, en la medida en que ésta se define como tal.

2. En ocasiones, es posible también que la decisión se sustente en la coincidencia de uno o más individuos con suficiente credibilidad para el clasificador, en cuanto a adscribir a otro a una categoría. En este caso, la decisión se toma sobre la base de una cierta forma de consenso social similar a los procedimientos clásicos de los estudios sobre estereotipos utilizados por ejemplo por Salazar y Marín (1977).

3. También puede ser posible que la decisión se sustente en lo que llamaremos el propio esquema inferencial del clasificador, es decir en un conjunto de creencias respecto de lo que él piensa que es necesario conocer de los demás para tomar una decisión orientada a otorgarles la pertenencia a una determinada categoría.

Como se observa, este esquema inferencial puede incluir la autodefinición del sujeto a clasificar, así como también el juicio coincidente de uno o más referentes confiables para el clasificador y el conjunto de creencias 
de este último, en cuanto a las características de las personas que él necesita conocer a fin de tomar una decisión clasificatoria.

El resultado de las exploraciones realizadas (Villegas, 1980) nos permite afirmar que las creencias pueden referirse a aspectos demográficos tales como el nivel educacional, de ingresos o de ocupación a manera de ejemplos. Pueden también ligarse a los aspectos conductuales como lo que la gente compra, usa o consume, los lugares que frecuenta, etc. Probablemente consideren también aspectos relativos a la conducta verbal sea en la forma o en el contenido o se refieran a características o rasgos de personalidad.

En las diferentes formas de interacción social, se clasifica a la gente como «simpáticas», «deportistas» o «de buena familia» si a juicio de quienes lo deciden cumplen con el requisito de poseer las características que permitan identificarlas como miembros de esas categorías. Es así como la percepción de similitud, de la posesión de algunas habilidades, del grado de contacto y familiaridad son factores que determinan una adscripción de las personas a la categoría «atractivas», según resume Santoro (1978).

Tanto la naturaleza, como el número y la amplitud de las categorías disponibles para la clasificación parecen ser muy altas. También la relevancia de las categorías en uso puede oscilar en función de variables asociadas a las personas involucradas, como de variables relativas al contexto: la «simpatía» en una reunión social puede ceder su lugar a la «inteligencia» o a la «experiencia» en ambientes científico-académicos de interacción.

En la investigación bibliográfica que hemos realizado, llama la atención un aparente predominio de categorías vinculadas a las características de personalidad, según se identifican coloquialmente. En este sentido pueden reportarse los datos de Ferreira y Rodrigues (1968) en los que observamos que de las siete principales características atribuidas a los estudiantes de Psicología por alumnos de otras carreras universitarias, por lo menos cuatro se refieren exclusivamente a elementos de la personalidad. (Tabla I).

\section{TABLA I}

Caracteristicas atribuidas con mayor frecuencia a los estudiantes de Psicología por sus compañeros de las otras carreras de la Pontificia Universidad Católica de Rio de Janeiro, según los datos de Ferreira y Rodrigues (1968)

\begin{tabular}{|c|c|}
\hline Caracteristicas & Frecuencia \\
\hline $\begin{array}{l}\text { Aproblemados } \\
\text { Investigadores } \\
\text { Idealistas } \\
\text { Observadores } \\
\text { Humanos } \\
\text { Interesados } \\
\text { Bien Intencionados }\end{array}$ & $\begin{array}{l}18 \\
18 \\
18 \\
17 \\
16 \\
14 \\
14\end{array}$ \\
\hline
\end{tabular}

NOTA: Las frecuencias aparentemente bajas para una muestra aleatoria sistemática de 60 sujetos se explican por el alto número de adjetivos que requieren los instrumentos clásicamente utilizados para este tipo de estudios.

En atención a estas sugerencias, un programa de investigaciones que se desarrolla en la Escuela de Psicología de la Pontificia Universidad Católica de Chile bajo la dirección del autor, tiene el propósito de examinar algunas variables para establecer la popularidad de los tipos de categorías utilizadas con mayor frecuencia para la clasificación social de las personas. 
Un ejemplo de investigación empírica en proceso

Como ya se había establecido, la investigación dedicada al examen de las estrategias utilizadas para la clasificación social de las personas contiene preferentemente referencias sobre categorías demográficas, conductuales y de personalidad. La comprobación de este hecho, nos orientó infructuosamente hacia la búsqueda de reportes e investigaciones que se refirieran de manera específica a un grupo de categorías difícilmente incorporadas a las anteriores y que sin embargo, pudiera relacionarse con todas ellas. Hablamos de aquellas dimensiones ligadas a los aspectos político-ideológicos presentes en las personas.

Los trabajos de Adorno, Frenkel-Brunswick, Levinson y Sanford (1950), o los de Eysenck (1964), pueden ubicarse dentro de los esfuerzos para la formulación de criterios «técnicos» utilizables en una identificación político-ideológica de las personas. En este último sentido, desconocemos investigaciones que analicen específicamente lo que ocurre en las diversas formas de interacción social diaria o cotidiana.

Los procesos de clasificación social de las personas en categorías político-ideológicas probablemente son menos amplios y menos frecuentes si se los compara con los señalados anteriormente. No obstante, bajo determinadas situaciones o circunstancias pueden y se han extendido a casi toda una población. Lo que estamos afirmando adquiere una significativa relevancia en la medida que no vemos razón alguna que permita suponer diferencias sustanciales entre la importancia psicosocial de estos procesos clasificatorios, con relación a los que se refieren a categorías demográficas, conductuales y de personalidad. No es raro que una vez que un sujeto ha sido adscrito a una categoría ideológico-política, puedan generarse expectativas para patrones considerados apropiados, aceptados o consecuentes. Tampoco es raro que la persona objeto de la clasificación experimente consecuencias derivadas de ésta, tanto en el plano afectivo-emocional como en el plano estrictamente conductual.

Tomando como base algunos de estos antecedentes, presentamos a continuación el resultado de un conjunto de exploraciones que forman parte de una investigación mayor.

Con el objeto de determinar las categorías más populares utilizadas en la clasificación política de las personas de una población restringida, un grupo de estudiantes de pregrado de la Universidad Central de Venezuela (UCV) fue distribuido heterogéneamente recurriendo al tipo de estudios, al sexo, al régimen y al nivel de la carrera. Por razones de economía y para el tipo de estudios se incluyeron sólo nueve de las 11 facultades que integran la Universidad. Las dos restantes tienen sus sedes fuera de la Ciudad Universitaria y en una ciudad relativamente distante de Caracas. Con respecto al régimen de estudios, los estudiantes de la UCV están en condiciones para escoger entre un horario diurno -con actividades entre las 8:00 $y$ las 15:00 horas- $y$ un horario nocturno - con actividades desde las 16:00 hasta las 22:00 horas-, lo cual sugiere importantes diferencias para estos dos sectores de la población estudiantil. El nivel de la carrera fue distribuido en tres estratos: el primero de ellos integrado por los estudiantes que cursaban $1 .^{\circ}, 2 .^{\circ}$ y $3 .^{\circ}$ semestre; el segundo por los estudiantes de $4 .^{\circ}, 5 .^{\circ}$ y $6 .^{\circ}$ semestre, y el tercero por aquellos estudiantes que hubieran superado estos niveles. 
La estructura de la muestra puede observarse en la tabla 2.

Los sujetos que integraban esta muestra fueron requeridos para que construyeran un listado de expresiones con las cuales ellos designaban a las personas que les eran políticamente afines o semejantes. Se les solicitó también la misma tarea pero en relación a las personas políticamente adversas, diferentes u opuestas.

\section{TABLA II}

Estructura de una muestra preliminar utilizada por Villegas (1980) para determinar las categorias politicas más frecuentes en la población estudiantil de la Universidad Central de Venezuela

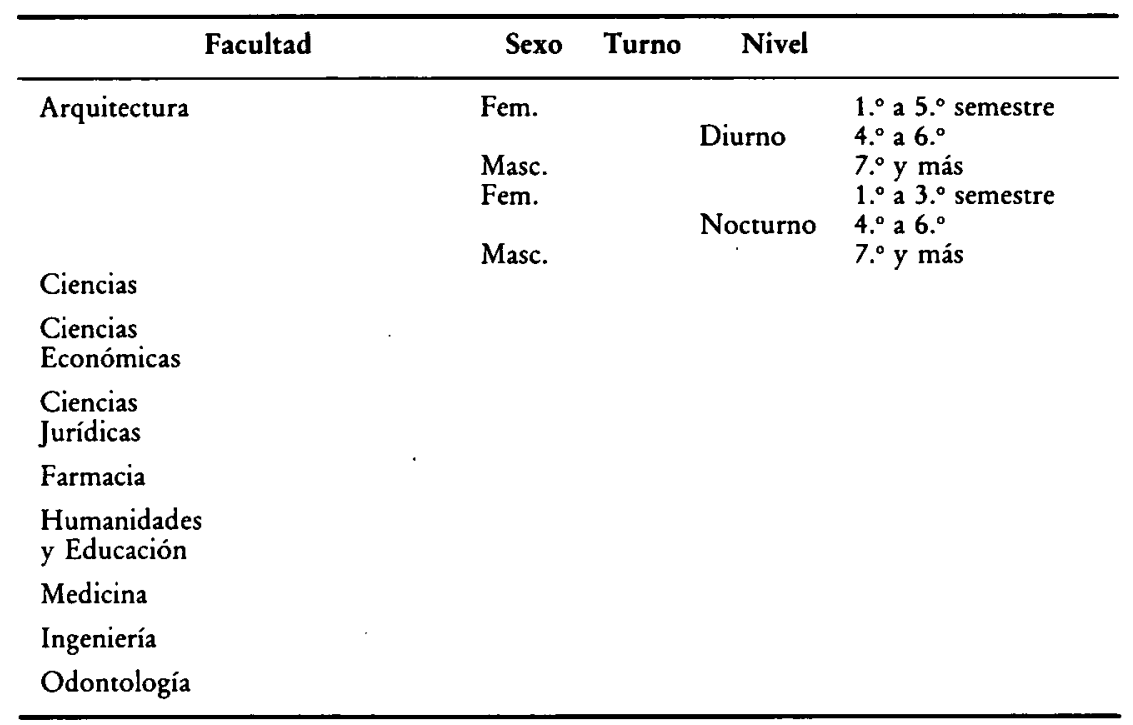

Los resultados obtenidos mostraron la distribución recogida en la tabla III.

El comentario de estos resultados, lo centraremos en las siguientes consideraciones:

1. En ausencia de reportes específicos que nos orientaran sobre el tema, recurrimos a los estudiantes universitarios bajo el supuesto de que, por lo menos en la mayoría de los países latinoamericanos, se lo caracteriza como uno de los sectores más inquietos desde el punto de vista político-ideológico. Esto mismo parece asegurar una cierta familiaridad con el lenguaje 'y la temática del instrumento aplicado:

2. Aunque las instrucciones fueron cuidadosamente formuladas, hay razones para asegurar que de manera indirecta se obtuvo datos sobre la autoclasificación político-ideológica de los propios sujetos.

3. Parece sugerente observar que las categorías mencionadas más frecuentemente por nuestros sujetos se refieren a tendencias generales y no a los partidos políticos venezolanos que, por cierto, son particularmente numerosos en el sector universitario estudiantil.

4. El contenido de la nota que acompaña a la tabla III confirmó nuestra suposición en el sentido de que para la clasificación política de las personas, parece conveniente considerar de manera especial la intervención de 
TABLA III

Categorias más utilizadas por los estudiantes de la Universidad Central de Venezuela (UCV), para la clasificación politica de las personas. Datos de Villegas (1980)

\begin{tabular}{llll}
\hline \multicolumn{1}{c}{$\begin{array}{c}\text { Personas } \\
\text { afines }\end{array}$} & (f) & \multicolumn{1}{c}{$\begin{array}{c}\text { Personas } \\
\text { adversas }\end{array}$} & (f) \\
\hline Izquierdistas & 36 & Reaccionarias & 40 \\
Revolucionarias & 32 & Demagogas & 39 \\
Progresistas & 30 & Derechistas & 26 \\
Independientes & 25 & Izquierdistas & 21 \\
Demócratas & 25 & Fascistas & 20 \\
Socialistas & 20 & Socialistas & 16 \\
Nacionalistas & 19 & Revolucionarias & 16 \\
Derechistas & 15 & Imperialistas & 14 \\
\hline
\end{tabular}

NOTA: Las respuestas incluyeron también categorias irreproductibles y con escasas o ninguna connotación política-ideológicas.

elementos altamente emocionales en la línea de atracción o rechazo.

5. Lo afirmado en el punto anterior puede observarse gráficamente la tabla IV.

\section{TABLA IV}

Juicio evaluativo de las categorias politicas utilizadas por un grupo de estudiantes de la Universidad Central de Venezuela (UCV), segun la inferencia de los datos de Villegas (1980)

\begin{tabular}{lll}
\hline $\begin{array}{c}\text { Evaluación } \\
\text { positiva }\end{array}$ & \multicolumn{1}{c}{$\begin{array}{c}\text { Evaluación } \\
\text { negativa }\end{array}$} & \multicolumn{1}{c}{$\begin{array}{c}\text { Evaluación } \\
\text { doble }\end{array}$} \\
\hline Progresistas & Reaccionarias & Derechistas \\
Demócratas & Demagogas & Izquierdistas \\
Independientes & Fascistas & Socialistas \\
Nacionalistas & Imperialistas & Revolucionarias \\
\hline
\end{tabular}

6. Interesa de manera particular el valor claramente positivo otorgado a la categoria NACIONALISTA, situación probablemente relacionada con un espíritu crítico de estos sujetos frente a la existencia de grupos de personas en Venezuela con una alta ideología dependiente de los Estados Unidos - IDUSA - expresión acuñada por Salazar (1979). En una investigación sobre creencias, actitudes nacionales y dependencia, este investigador preguntaba por ejemplo:

¿Cuál es el país más desarrollado?

¿Cuál es el país más organizado?

¿Cuál es el país de gente más trabajadora?

¿Cuál es el país de gente más inteligente?

¿Cuál es el país de gente má capaz?

Las respuestas de un grupo de sujetos que señalaban preferentemente a los Estados Unidos, fueron descritos como típicas de las personas con una alta IDUSA.

Todas las consideraciones y experiencias recogidas en nuestro trabajo, nos impulsan a continuar las investigaciones y a sugerir su extensión a diferentes países, con el propósito de establecer tanto las diferencias, como las semejanzas que tuvieron lugar. 


\section{Referencias}

Adorno, T.; ERenkel-Brunswick; LeVinson, D. J., y SANDFord, R. R. (1950): The Autboritarian Personality New York: Harper and Row.

AlLPORT, G. W. (1954): The Nature of Prejudice Cambridge: Addisson Press.

ANDERSON, H. (1965): "Averaging versus Addings as Stimulus Combination Rules in Impression Formation*. Journal of Experimental Psychology 70:394-400.

ARKIN, R. H., y Duval, S. (1975). «Focus of Attention and Causal Attributions of Actors and Observersm. Journal of Experimental Social Psychology, 11:427-438.

AsCH, S. (1946). *Forming Impressions of Personality». Journal of Abnormal and Social Psychology, 41:258-290.

Baron, R.; GRIFFITT, W., y BYRNe, D. (1974). Social Psychology: Understanding Human Interaction, New York: Allyn Bacon.

Bandura, A. (1973). Agression: A Social Learning Analysis. New Jersey: Prentice Hall.

BECKER, H. (1974). «Labelling Theory Reconsidered». En: P. Rock y M. McIntocsh (Eds.) Deviance and Social Control, London: Tavistock.

BERKOWITZ, L. (1974). «Some Determinants of Impulsive Aggression: Role of Mediated Associations with Reinforcements of Aggression». Psychological Review, 81:165-176.

FishBEIN, M. (1979). Comunicacion personal en Seminario de Investigación. Universidad Central de Venezuela: Caracas.

Friedland, S. J.; CrocketT, W. M., y Laird, J. D. (1973). *The Effects of Role and Sex on the Perception of Others». Journal of Social Psychology, 91:273-283.

JAHODA, G. (1956). "Political Attitudes and Judgments of Other People*. Journal of Abnormal and Social Psychology 49.

JONES, E., y ARONSON, E. (1973). *Attribution of Fault to a Rape Victim as a Function of Respectability of the Victimn. Journal of Personality and Social Psychology, 26:415-419.

Jones, E., y Davis, K. (1965). «From Acts to Dispositions: The Attribution Process in Persons Perception». En: L. Berkowitz (Ed.), Advances in Experimental Social Psychology New York: Academic Press.

Kelley, H. H. (1979). Personal relationships: Their Structures and Processes, Hillsdale, N. J.: L. Erlbaum.

Kelley, H. H. (1967) «Attribution Theory in Social Psychology». En: D. Lavin (Ed.), Nebraska Symposium on Motivation, Lincoln: Univ. of Nebraska Press.

Kelly, G. (1959). The Psychology of Personal Constructs, New York: Norton.

Kleck, B.; ONO, R., y HastoRFF, A. H. (1966). *The Effects of Physical Deviance upon Face to Face Interaction. Human Relations, 19.

Kruglansky, A. (1975). "The Endogenous - Exogenous Partition in Attribution Theory". Psychological Review, S2:387-406.

Lerner, M. (1971). «Observer Evaluation of a Victim: Justice, Guilt and Veridical Perception.. Journal of Personality and Social Psychology, 20.

Livesley, W., y BROMLEY, D. (1973). Person Perception in Childhood and Adolescence, London: Free Press.

NisBeTt, R., y BoRgida, E. 1. (1973). "Attribution in the Psychology of Prediction». Joumal of Personality and Social Psychology, 32.

ORANTES, A. (1979). Dimensiones de la evaluación y factores subjetivos. Presentación en las II Jornadas de Investigación Educativa. Escuela de Educación. Universidad Central de Venezuela. Caracas, 1979.

Rodrigues, A. (1979). Estudos em Psicologia Social. Petropolis: Vozes.

RoDrigues, A., y FERreira, M. (1968). «Estereotipos em Relacao a Alunos de Psicologia num Campus Universitarion. Arquivos Brasileiros de Psicologia Aplicada, 20:9-20.

RoKeach, M., y ROTHMAN, G. (1965). "The principle of Belief Congruence and the Congruity Principle as Models of Cognitive Interaction w. Psychological Review, 72.

RommetverT, R. (1960). Selectivity, Intuition and Halo Effects in Social Perception. Oslo: Oslo University Press.

ROSENTHAL, R., y JACOBSON, L. F. (1968). «Teacher's Expectations for Disadvantaged». Scientific American, 218:4.

SANTORO, E. (1978). «Percepción Social». En J. M. Salazar (Ed.): Psicología Social, México: Trillas.

SalazAR, J. M. (1979). Creencias, Actitudes Nacionales y Dependencia. Conferencia en el Congreso Interamericano de Psicología, Lima.

SARBIN, T.; TAFT, R., y Balley, D. (1960). Clinical Inference and Cognitive Theory, New York: Holt, Rinehart and Winston.

SECORD, P., y BACKMAN, C. (1964). Social Psychology, New York: McGraw-Hill.

SCHNEIDER, D. (1973). «Implicit Personality Theory: A Review». Psychological Bulletin, 79:294-309.

Tagiuri, R. (1969). «Person Perception». En G. Lindzey y E. Aronson (Eds.), The Handbook of Social Psychology, Reading: Addisson-Wesley. 
Shrauger, S. (1967). «Cognitive Differentiation and the Impression Formation Process». Journal of Personality, 35 .

TAJFEL, H. (1969). «Social and Cultural Factors in Perception*. En G. Lindzey y E. Aronson (Eds.), Handbook of Social Psychology, Reading: Addison-Wesley.

VILLEGAS, J. F. (1980). Etiquetamiento politico. Universidad Central de Venezuela (no publicado).

Wark, P., y.Knapper, C. (1963): The Perception of People and Events, New York: Wiley.

WILLES, R. M. (1960). "Stimulus Pooling and Social Perception*. Journal of Abnormal and Social Psychology, 60:365-373.

WISHNER, J. (1960). "Reanalysis of Impressions of Personality». Psychological Review, 67:96-112. 